\title{
Produtividade e bienalidade da produção de cafezais adensados, sob diferentes doses de $\mathbf{N}$ e K
}

\author{
Samuel Vasconcelos Valadares(1), Júlio César Lima Neves ${ }^{(1)}$, Gustavo Nogueira Guedes Pereira Rosa(2), \\ Hermínia Emília Prieto Martinez ${ }^{(3)}$, Víctor Hugo Alvarez Venegas ${ }^{(1)}$ e Paulo César de Lima ${ }^{(4)}$
}

(1)Universidade Federal de Viçosa (UFV), Departamento de Solos, Avenida P.H. Rolfs, s/no, Campus Universitário, CEP 36570-000 Viçosa, MG. E-mail: samuel.valadares@ufv.br, julio_n2003@yahoo.com.br, vhav@ufv.br (2)Fertilizantes Heringer, BR 262, Km 39,4, Ponte da Aldeia, CEP 36900-000 Manhuaçu, MG. E-mail: gustavongpr@hotmail.com (3)UFV, Departamento de Fitotecnia. E-mail: hermínia@ufv.br ${ }^{(4)}$ Empresa de Pesquisa Agropecuária de Minas Gerais, Centro de Tecnologias da Zona da Mata, Vila Gianette, Casa 46, CEP 36570-000 Viçosa, MG. E-mail: plima@epamig.br

Resumo - O objetivo deste trabalho foi avaliar o efeito da adubação com N e K sobre a produtividade e a bienalidade de produção de cafeeiros em plantio adensado, em estudo de longa duração. Foram avaliados dois experimentos, na região da Zona da Mata de Minas Gerais, conduzidos em blocos ao acaso, com parcelas subdivididas no tempo. No primeiro experimento, realizado no espaçamento de $1,5 \mathrm{x} 0,7 \mathrm{~m}$ (9.523 plantas por hectare), foram avaliados os efeitos de sete doses de $\mathrm{N}+\mathrm{K}_{2} \mathrm{O}$ na proporção 1:1 $(0,200,400,600,800,1.000$ e $1.200 \mathrm{~kg} \mathrm{ha}^{-1}$ por ano), durante oito safras (2001/2002 a 2008/2009). Como o fertilizante utilizado tinha proporção 1:1, para obter a dose utilizada de cada elemento $\left(\mathrm{N}\right.$ ou $\left.\mathrm{K}_{2} \mathrm{O}\right)$, deve-se dividir a dose total por dois. $\mathrm{O}$ segundo experimento foi conduzido no espaçamento de 2,5x0,6 $\mathrm{m}$ (6.666 plantas por hectare), em arranjo fatorial, com cinco doses de $\mathrm{N}\left(0,150,300,450\right.$ e $600 \mathrm{~kg} \mathrm{ha}^{-1}$ por ano) e cinco doses de $\mathrm{K}_{2} \mathrm{O}(0,150,300,450 \mathrm{e}$ $600 \mathrm{~kg} \mathrm{ha}^{-1}$ por ano), durante seis safras (2005/2006 a 2010/2011). As doses associadas à máxima produtividade de café beneficiado variaram de 424 a $560 \mathrm{~kg} \mathrm{ha}^{-1}$, para $\mathrm{N}$, e de 21 a $338 \mathrm{~kg} \mathrm{ha}^{-1}$, para $\mathrm{K}_{2} \mathrm{O}$. A adubação com N reduz os efeitos da bienalidade da produção do cafeeiro, e a adubação com $\mathrm{K}$, em anos de baixa produtividade, favorece a recuperação da produtividade do cafeeiro no ano seguinte.

Termos para indexação: Coffea arabica, estabilidade produtiva, fertilização, nutrição mineral.

\section{Yield and production bienniality of dense coffee plantations under different levels of $\mathbf{N}$ and $\mathrm{K}$}

\begin{abstract}
The objective of this work was to evaluate the effect of $\mathrm{N}$ and $\mathrm{K}$ fertilization on the yield and production bienniality of dense coffee plantations, in a long-term study. Two experiments were evaluated in the Zona da Mata region of Minas Gerais, Brazil, under a randomized complete block design, split-plotted in time. In the first experiment, carried out with $1.5 \times 0.7 \mathrm{~m}$ spacing (9,523 plants per hectare), the effects of seven doses of $\mathrm{N}+\mathrm{K}_{2} \mathrm{O}$ in the proportion of 1:1 were evaluated $\left(0,200,400,600,800,1,000\right.$, and 1,200 $\mathrm{kg}^{-1}$ per year) during eight crop seasons (2001/2002 to 2008/2009). Since the fertilizer had 1:1 proportion, in order to obtain the dose for each nutrient $\left(\mathrm{N}\right.$ or $\left.\mathrm{K}_{2} \mathrm{O}\right)$, the total dose must be divided by two. The second experiment was done with $2.5 \times 0.6$ spacing (6,666 plants per hectare), under a factorial arrangement, with five doses of $\mathrm{N}$ $\left(0,150,300,450\right.$, and $600 \mathrm{~kg} \mathrm{ha}^{-1}$ per year $)$ and five doses of $\mathrm{K}_{2} \mathrm{O}\left(0,150,300,450\right.$, and $600 \mathrm{~kg} \mathrm{ha}^{-1}$ per year), during six crop seasons (2005/2006 to 2010/2011). The doses related to the maximum yield of processed coffee ranged from 424 to $560 \mathrm{~kg} \mathrm{ha}^{-1}$, for $\mathrm{N}$, and from 21 to $338 \mathrm{~kg} \mathrm{ha}^{-1}$, for $\mathrm{K}_{2} \mathrm{O}$. Nitrogen fertilization reduces the effect of production bienniality of coffee plants, and $\mathrm{K}_{2} \mathrm{O}$ fertilization, in years of low productivity, promotes yield recovery of coffee plants in the next year.
\end{abstract}

Index terms: Coffea arabica, production stability, fertilization, mineral nutrition.

\section{Introdução}

O adensamento de plantio proporciona considerável impacto econômico na cafeicultura (Pereira et al., 2011). O sistema adensado, caracterizado por densidades populacionais entre 5.000 e 10.000 plantas por hectare, se devidamente empregado, permite aumentar a produtividade de grãos e melhorar a eficiência de utilização da mão-de-obra e de insumos, o que reduz o tempo de retorno do investimento, diminui o custo por saca e aumenta a lucratividade da atividade. Entretanto, para a adoção do sistema adensado, são requeridas alterações sistemáticas no 
manejo da lavoura, especialmente no manejo de podas, na irrigação e na fertilização.

A maior competição entre plantas, com o aumento da densidade de plantio, resulta em alterações fisiológicas e morfológicas associadas à diminuição da produção por planta e ao aumento da produção por área, tanto para o crescimento vegetativo como para a produção de frutos. O adensamento proporciona melhor aproveitamento da radiação solar e recuperação dos nutrientes, em decorrência das maiores superfície foliar e densidade radicular (Da Mata \& Rena, 2002; Prezotti \& Rocha, 2004). Portanto, com a redução do espaçamento, as exigências nutricionais são alteradas. Pesquisas têm mostrado que estas exigências não crescem proporcionalmente ao aumento da população de plantas, e que a extrapolação das doses aplicadas por cova em plantios convencionais para o sistema adensado normalmente resulta em aplicação de quantidades inadequadas de fertilizantes (Gallo et al., 1999; Augusto et al., 2007; Sobreira et al., 2011). Além disso, a alteração no espaçamento de plantio pode modificar o ciclo bienal do cafeeiro (Pereira et al., 2011).

É grande o interesse na avaliação da resposta de cafeeiros adensados à adubação com $\mathrm{N}$ e K, em regiões produtoras (Pavan et al., 1994; Figueiredo et al., 2006; Prezotti \& Rocha, 2004; Bragança et al., 2009). Entretanto, os estudos têm sido conduzidos com poucas safras e normalmente não contemplam o efeito da adubação sobre a bienalidade da produção.

O objetivo deste trabalho foi avaliar o efeito da adubação com $\mathrm{N}$ e $\mathrm{K}$ sobre a produtividade e a bienalidade de produção de cafeeiros em plantio adensado, em estudo de longa duração.

\section{Material e Métodos}

Foram realizados dois experimentos de campo, no Centro de Pesquisas Cafeeiras Eloy Carlos Heringer, localizado no Município de Martins Soares, MG ( $20^{\circ} 14^{\prime} 45^{\prime \prime} \mathrm{S}$ e $41^{\circ} 50^{\prime} 47^{\prime \prime} \mathrm{W}$, a $763 \mathrm{~m}$ de altitude). O clima da região, segundo a classificação de Köppen, é do tipo Cwa, mesotérmico com verões úmidos. O solo da área experimental é um Latossolo Vermelho-Amarelo distrófico (Santos et al., 2006), cujas características químicas são apresentadas na Tabela 1. As amostras de solo foram coletadas de acordo com o método proposto por Cantarutti et al. (1999).

Oplantio foirealizadoem covas de $0,40 \mathrm{x} 0,40 \mathrm{x} 0,20 \mathrm{~m}$, e as adubações de plantio e a formação dos cafeeiros foram baseadas nas recomendações propostas por Guimarães et al. (1999), conforme os resultados da análise química do solo.

A bienalidade da produção foi avaliada por meio de diferentes abordagens. A primeira baseou-se no cálculo dos coeficientes de variação dos dados de produtividade em cada parcela, de modo a se ter ideia da variabilidade média entre safras consecutivas. $\mathrm{Na}$ segunda abordagem, a bienalidade foi avaliada entre anos consecutivos, com uso das seguintes equações: bienalidade nos anos de alta para baixa produtividade $(\%)=[(\mathrm{Pa}-\mathrm{Pb}) / \mathrm{Pa}] \times 100$, em que: $\mathrm{Pa}$ é a produtividade no ano de alta e $\mathrm{Pb}$ é a produtividade no ano de baixa; e bienalidade nos anos de baixa para alta produtividade $(\%)=[(\mathrm{Pa}-\mathrm{Pb}) / \mathrm{Pb}] \times 100$.

O experimento 1 foi implantado em dezembro de 1993, com cafeeiros da variedade Catuaí Vermelho 44, no espaçamento de $1,5 \times 0,7 \mathrm{~m}$ (9.523 plantas por hectare), e finalizado na safra 2008/2009. Os tratamentos foram compostos por sete doses de $\mathrm{N}+\mathrm{K}_{2} \mathrm{O}$, na proporção 1:1: 0, 200, 400, 600, 800, 1.000 e $1.200 \mathrm{~kg} \mathrm{ha}^{-1}$ por ano. Como foi utilizada a proporção $1: 1$, dividiu-se a dose total por dois para obter a dose utilizada de cada elemento $\left(\mathrm{N}\right.$ ou $\left.\mathrm{K}_{2} \mathrm{O}\right)$. A unidade experimental foi constituída por três linhas de nove plantas, e a parcela útil, formada pelas sete plantas centrais. Utilizou-se o delineamento de blocos ao acaso, com quatro repetições, no arranjo de parcelas subdivididas no tempo. As adubações com N e K tiveram, como fonte, a formulação N-P-K (20-00-20) e foram parceladas em três aplicações anuais, cada uma equivalente a $1 / 3$ da dose total, realizadas em outubro, dezembro e março. Foram avaliados os dados de produtividade e bienalidade (avaliada com ambos os métodos) obtidos nos anos agrícolas 2001/2002,

Tabela 1. Características químicas dos solos utilizados, antes da aplicação dos tratamentos.

\begin{tabular}{|c|c|c|c|c|c|c|c|c|c|}
\hline Experimento & $\begin{array}{c}\mathrm{pH} \\
\left(\mathrm{H}_{2} \mathrm{O}\right)\end{array}$ & $\mathrm{P}$ & $\begin{array}{c}\mathrm{K}^{+} \\
\left.\mathrm{I}^{-3}\right)---- \\
\end{array}$ & $\mathrm{Ca}^{2+}$ & $\mathrm{Mg}^{2+}$ & $\mathrm{Al}^{3+}$ & $\mathrm{H}+\mathrm{Al}$ & $\begin{array}{c}\text { MO } \\
\left(\operatorname{dag~kg}^{-1}\right)\end{array}$ & $\begin{array}{c}\text { P-rem } \\
\left(\mathrm{mg} \mathrm{L}^{-1}\right)\end{array}$ \\
\hline 1 & 6,0 & 8,1 & 24 & 4,6 & 1,3 & 0,0 & 5,3 & 3,6 & 23,6 \\
\hline 2 & 4,7 & 4,3 & 132 & 0,5 & 0,2 & 0,9 & 5,9 & 3,7 & 18,1 \\
\hline
\end{tabular}


$2002 / 2003, \quad 2003 / 2004, \quad 2004 / 2005, \quad 2005 / 2006$, 2006/2007, 2007/2008 e 2008/2009.

No experimento 2, procurou-se determinar os efeitos isolados de $\mathrm{N}$ e de $\mathrm{K}$, bem como avaliar a interação entre esses nutrientes. $\mathrm{O}$ experimento foi instalado em novembro de 2003, com cafeeiros da variedade Catucaí Amarelo 6/30, no espaçamento de 2,5x0,6 m (6.666 plantas por hectare). Utilizou-se o delineamento de blocos ao acaso, em arranjo fatorial, com três repetições e parcelas subdivididas no tempo. Foram avaliadas cinco doses de $\mathrm{N}(0,150,300,450 \mathrm{e}$ $600 \mathrm{~kg} \mathrm{ha}^{-1}$ por ano) e cinco doses de $\mathrm{K}_{2} \mathrm{O}(0,150,300$, 450 e $600 \mathrm{~kg} \mathrm{ha}^{-1}$ por ano). A unidade experimental foi constituída por três linhas de sete plantas, sendo a parcela útil composta pelas cinco plantas centrais. As doses de $\mathrm{N}$ foram fornecidas na forma de ureia, e as de $\mathrm{K}$, na de $\mathrm{KCl}$. As doses foram parceladas em três aplicações anuais, sem incorporação, sob a copa das plantas, cada uma equivalente a $1 / 3$ da dose total, em outubro, dezembro e março. Foram avaliados os dados de produtividade e bienalidade obtidos ao longo de seis safras: 2005/2006, 2006/2007, 2007/2008, 2008/2009, $2009 / 2010$ e 2010/2011. Também foram avaliados os teores de $\mathrm{N}$ e $\mathrm{K}$ no tecido foliar.

A análise do tecido foliar foi realizada na safra 2010/2011, no experimento 2. A amostragem das folhas foi feita após a colheita, tendo-se coletado o terceiro ou o quarto par de folhas a partir do ápice do ramo, à meia altura das plantas, num total de 30 folhas por unidade experimental. Foram quantificados os teores de $\mathrm{N}$ pelo método Kjeldahl, e os de $\mathrm{K}$, por fotometria de emissão de chama, nos extratos da mineralização nítrico-perclórica.

Os dados de produtividade, bienalidade e de teores foliares de $\mathrm{N}$ e $\mathrm{K}$ foram submetidos à análise de variância (Anova) e de regressão. As doses para obtenção da produtividade máxima foram calculadas igualando-se a derivada primeira das equações de regressão a zero.

\section{Resultados e Discussão}

No experimento 1, a adubação com $\mathrm{N}$ e K aumentou a produção em todas as safras, com exceção de 2003/2004 (Tabela 2). A média das oito safras avaliadas também foi afetada pela adubação. As doses de $\mathrm{N}+\mathrm{K}_{2} \mathrm{O}(1: 1)$ para obtenção da produtividade máxima variaram de 660 a $999 \mathrm{~kg} \mathrm{ha}^{-1}$ dos nutrientes na proporção 1:1. Essas doses correspondem às de 330 a $499 \mathrm{~kg} \mathrm{ha}^{-1}$ de cada nutriente (dose total dividida por dois). Os baixos teores de K do solo (Tabela 1), bem como com o elevado requerimento nutricional da cultura por estes nutrientes (Laviola et al., 2006) justificam a resposta observada à adubação.

Figueiredo et al. (2006), com base em três ensaios no sul de Minas Gerais, recomendam doses menores do que as doses ótimas obtidas no presente trabalho. Entretanto, nesses ensaios, as produtividades obtidas foram menores e os teores de $\mathrm{K}$ no solo foram maiores que os observados no presente trabalho. Outra diferença importante entre os estudos refere-se à população de plantas utilizada, que altera a resposta da cultura à fertilização (Braccini et al., 2002; Prezotti \& Rocha, 2004). Embora ambos os trabalhos tenham sido realizados com cafeeiros adensados, no experimento no sul de Minas, a população de plantas era menor (6.667 plantas por hectare).

Apesar de o experimento 1 ter mostrado que o manejo da adubação com $\mathrm{N}$ e $\mathrm{K}$ pode aumentar consideravelmente a produtividade de cafeeiros adensados, o experimento não é conclusivo quanto aos efeitos isolados dos dois nutrientes nem quanto a suas interações. Neste particular, é importante ressaltar que o requerimento nutricional do cafeeiro por $\mathrm{N}$ não deve ser, necessariamente, o mesmo que o por $\mathrm{K}$ (Neves et al., 2006). Além disso, o comportamento das formas disponíveis desses elementos no solo é diferente, o que também influencia a resposta à adubação (Römheld \& Kirkby, 2010; Tsay et al., 2011; Souza et al., 2012).

$\mathrm{O}$ experimento 2, conduzido em arranjo fatorial, é mais adequado para caracterizar os efeitos isolados

Tabela 2. Produtividade de café (sacas por hectare) em função de doses de $\mathrm{N}+\mathrm{K}\left(\mathrm{kg} \mathrm{ha}^{-1}\right.$ de $\mathrm{N}+\mathrm{K}_{2} \mathrm{O}$, aplicados na proporção $1: 1)$.

\begin{tabular}{llccc}
\hline Safra & \multicolumn{1}{c}{ Equação } & $\mathrm{R}^{2}$ & $\begin{array}{c}\text { Dose para } \\
\text { MET }\end{array}$ & $\begin{array}{c}\text { Produtividade } \\
\text { máxima }\end{array}$ \\
\hline $2002 / 2003$ & $\hat{y}=24,1647+0,1649 * * \mathrm{x}-0,00008 \mathrm{x}^{2}$ & 0,95 & 999,4 & 109,1 \\
$2003 / 2004$ & $\hat{\mathrm{y}}=14,07$ & - & - & - \\
$2004 / 2005$ & $\hat{y}=6,84+0,146 * * \mathrm{x}-0,000075 * * \mathrm{x}^{2}$ & 0,96 & 973,3 & 77,9 \\
$2005 / 2006$ & $\hat{\mathrm{y}}=2,98+3,051 * * \mathrm{x}^{0,5}-0,058^{*} \mathrm{x}$ & 0,74 & 691,8 & 43,1 \\
$2006 / 2007$ & $\hat{\mathrm{y}}=18,50+0,093 * * \mathrm{x}-0,00005^{*} \mathrm{x}^{2}$ & 0,68 & 930,0 & 61,7 \\
$2007 / 2008$ & $\hat{\mathrm{y}}=1,85+0,167 * * \mathrm{x}-0,0001 * * \mathrm{x}^{2}$ & 0,96 & 835,0 & 67,9 \\
$2008 / 2009$ & $\hat{\mathrm{y}}=17,45+5,806 * * \mathrm{x}^{0,5}-0,113 * * \mathrm{x}$ & 0,80 & 660,0 & 92,0 \\
$2009 / 2010$ & $\hat{y}=3,93+0,067 * * \mathrm{x}-0,000035^{*} \mathrm{x}^{2}$ & 0,77 & 957,2 & 36,0 \\
\hline Média & $\hat{\mathrm{y}}=16,05+0,1058^{* *} \mathrm{x}-0,00006^{*} \mathrm{x}^{2}$ & 0,93 & 881,7 & 61,9 \\
\hline
\end{tabular}

* e **Significativo a 5 e $1 \%$ de probabilidade, respectivamente. MET: máxima eficiência técnica. 
de cada nutriente e da interação entre eles. Neste experimento, as doses de $\mathrm{N}$ e $\mathrm{K}$ e suas interações somente não influenciaram a produtividade do cafeeiro nas duas primeiras safras avaliadas (Figura 1).

A exigência do cafeeiro por $\mathrm{N}$ foi maior que a exigência por $\mathrm{K}$. As doses de $\mathrm{N}$ e $\mathrm{K}_{2} \mathrm{O}$ associadas à máxima produtividade de café beneficiado, nas safras em que houve resposta à fertilização, foram, respectivamente, de: 424 e $373 \mathrm{~kg} \mathrm{ha}^{-1}$, na safra 3; 388,8 e $21,4 \mathrm{~kg} \mathrm{ha}^{-1}$, na safra $4 ; 534,9$ e $268,6 \mathrm{~kg} \mathrm{ha}^{-1}$, na safra 5; e 560 e $337,9 \mathrm{~kg} \mathrm{ha}^{-1}$, na safra 6 .

Embora, segundo Alvarez V. et al. (1999), o teor inicial de $\mathrm{K}$ no solo (Tabela 1 ) tenha sido elevado - o que explicaria parcialmente a menor resposta à adição do elemento -, os resultados obtidos estão de acordo com os de diversos trabalhos, que relatam elevado
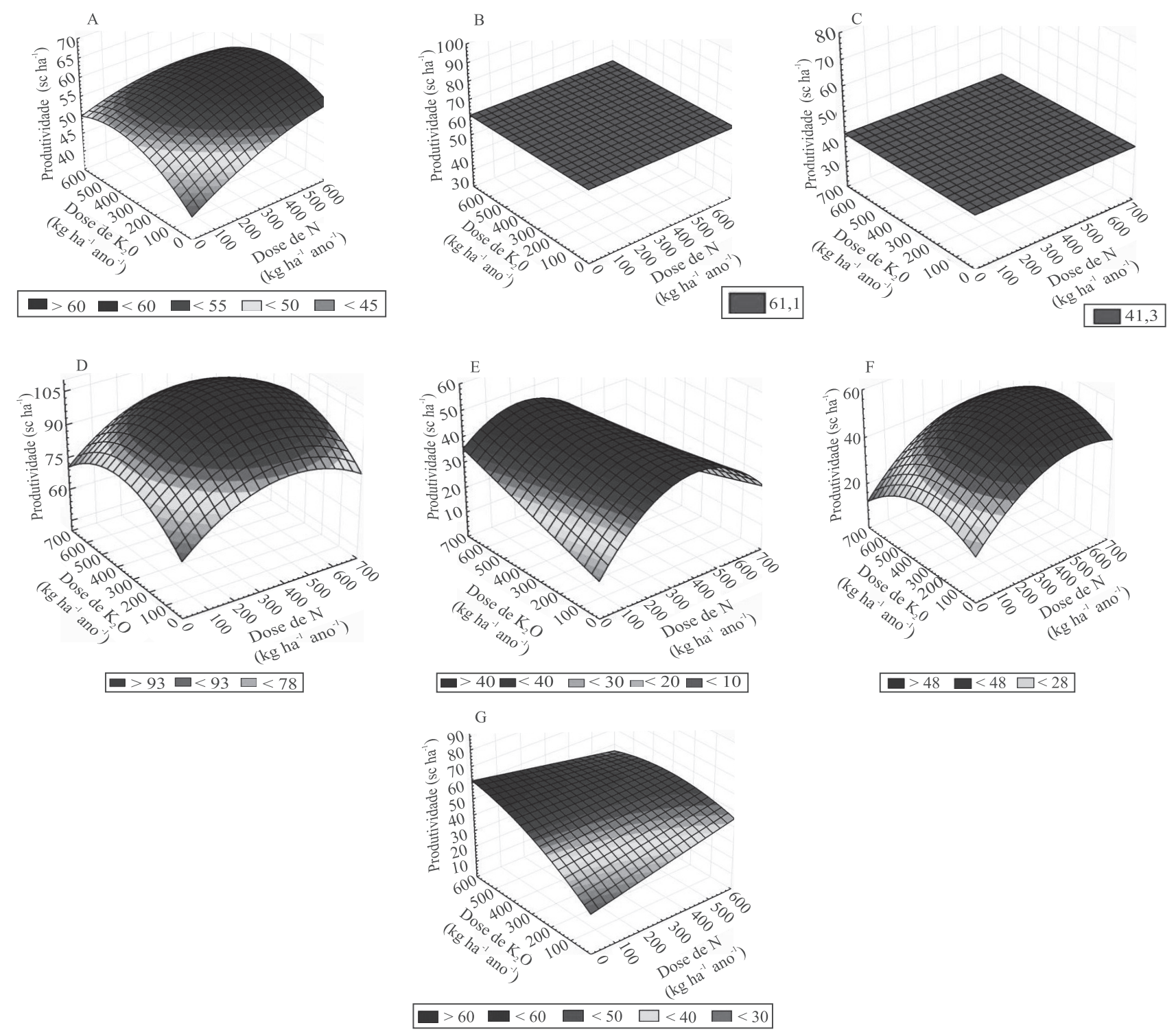

Figura 1. Produtividade do cafeeiro em sistema adensado de plantio em função de doses de $\mathrm{N}$ e $\mathrm{K}$ ao longo de seis safras agrícolas (experimento 2): A, produtividade média das seis safras: $\hat{\mathrm{y}}=41,23+0,04 * * \mathrm{~N}-0,000026^{*} \mathrm{~N}^{2}+0,051^{*} \mathrm{~K}-0,000062^{\circ} \mathrm{K}^{2}$ - $0,000029^{+} \mathrm{NK}\left(\mathrm{R}^{2}=0,44\right) ; \mathrm{B}$, produtividade da safra $1: \hat{\mathrm{y}}=\overline{\mathrm{y}}=61,1$ sacas por hectare; $\mathrm{C}$, produtividade da safra $2: \hat{\mathrm{y}}=\overline{\mathrm{y}}=41,3$ sacas por hectare; $\mathrm{D}$, produtividade da safra $3: \hat{\mathrm{y}}=66,37+0,1087 * * \mathrm{~N}-0,000128^{*} \mathrm{~N}^{2}+0,09443^{\circ} \mathrm{K}-0,0001266^{*} \mathrm{~K}^{2}\left(\mathrm{R}^{2}=0,48\right)$; E, produtividade da safra $4: \hat{y}=14,88+0,1369 \mathrm{~N}+0,02908 \mathrm{~K}-0,000174 * * \mathrm{~N}^{2}-0,0000748^{+} \mathrm{NK}\left(\mathrm{R}^{2}=0,24\right)$; F, produtividade da safra 5: $\hat{y}=20,02+0,0989^{* *} \mathrm{~N}+0,05128 \mathrm{~K}-0,00009248^{\circ} \mathrm{N}^{2}-0,000089^{+} \mathrm{K}^{2}\left(\mathrm{R}^{2}=0,43\right)$; e $\mathrm{G}$, produtividade da safra 6 : $\hat{\mathrm{y}}=25,29+0,033 \mathrm{~N}+0,102 * * \mathrm{~K}-0,00007^{+} \mathrm{K}^{2}-0,00009765^{*} \mathrm{NK}\left(\mathrm{R}^{2}=0,20\right) . * *,{ }^{*},{ }^{\circ},{ }^{+}$Significativo a $1,5,10$ e $15 \%$ de probabilidade, respectivamente. 
requerimento do cafeeiro por $\mathrm{N}$, nutriente comumente associado a maiores ganhos na produtividade da cultura pela adubação (Prezotti \& Rocha, 2004; Figueiredo et al., 2006). Neves et al. (2006), em trabalho com quatro variedades de café e dois níveis de adubação, obtiveram relações médias entre $\mathrm{N}$ e $\mathrm{K}$ acumulados equivalentes a 2,36 e 1,51, aos 31 e aos 55 meses, respectivamente. Esses resultados são indicativos de que o emprego generalizado de formulações como 20-05-20 e 20-00-20, para cafezais em produção, comuns na cafeicultura, pode estar relacionado a fertilizações desequilibradas e ao desperdício de fertilizantes.

Independentemente da produção, o requerimento de $\mathrm{N}$ foi crescente até o sexto ano, ao passo que o requerimento de $\mathrm{K}$, pelo menos até o quinto ano de produção, acompanhou as variações de produtividade ocorridas em cada ano e, portanto, esteve associado ao ciclo bienal do cafeeiro. Este resultado está de acordo com as curvas de acúmulo e partição de nutrientes para a cultura (Laviola et al., 2006). A acumulação de N normalmente acompanha o incremento de biomassa da lavoura (Neves et al., 2006), o que significa que a exigência nutricional, em cafeeiros adensados, assim como no espaçamento convencional, varia pouco com a produtividade. Nos anos de baixa carga de frutos, os órgãos vegetativos substituem os frutos como drenos para o nutriente (Malavolta et al., 2002). O K, por sua vez, é mais requerido nos anos de alta produtividade, em razão de sua maior importância na fase reprodutiva da planta, por ser o macronutriente mais exportado nos frutos (Laviola et al., 2006). Contudo, é importante observar que, apesar de o $\mathrm{K}$ ser mais requerido nos anos de alta produtividade, as produções obtidas nesses anos podem ser influenciadas pelas adubações realizadas nos anos de baixa produtividade, como se discute a seguir.

$\mathrm{Na}$ sexta safra, que foi de baixa produtividade, a exigência das plantas por $\mathrm{K}$ aumentou em relação ao ano anterior, e o requerimento por $\mathrm{N}$ foi pouco maior que o obtido na quinta safra. Essa constatação é reforçada pelo fato de o coeficiente linear referente ao efeito do N não ter sido significativo nesse ano agrícola, diferentemente do observado nos outros anos. Nesta safra, houve apenas efeito das doses de K e da interação $\mathrm{N}$ e K (Figura 1). Este resultado possivelmente está associado ao maior fechamento do dossel da cultura verificado nessa safra, conforme indicaram Gallo et al.
(1999), que atribuíram as menores respostas, ou mesmo o efeitos depressivos da adubação nitrogenada sobre a produtividade do cafeeiro, ao excessivo adensamento das plantas em lavouras mais velhas.

Os teores foliares de $\mathrm{N}$ e $\mathrm{K}$, avaliados após a colheita, aumentaram em resposta às doses de $\mathrm{N}$ e $\mathrm{K}_{2} \mathrm{O}$ (Figura 2), o que era esperado e está de acordo com o aumento observado na produtividade.

Quanto à análise da bienalidade de produção, constatou-se que o coeficiente de variação de cada parcela foi menor nos tratamentos com maiores doses de $\mathrm{N}$ e $\mathrm{K}$, ao longo das oito safras avaliadas no experimento 1 , o que resultou em maior estabilidade produtiva do cafeeiro (Figura 3 ).

$\mathrm{Na}$ avaliação do efeito conjunto de $\mathrm{N}$ e $\mathrm{K}$ sobre a estabilidade da produção (experimento 1), dentro de cada biênio, a adição desses nutrientes esteve associada a reduções, em termos relativos, menos intensas da produtividade de frutos em três de quatro biênios, na transição dos anos de alta para os de baixa produtividade (Tabela 3). Na transição dos anos de baixa para os de alta produtividade, também verificouse aumento relativo menos acentuado, em dois dos três biênios. Portanto, o maior suprimento de $\mathrm{N}$ e $\mathrm{K}$
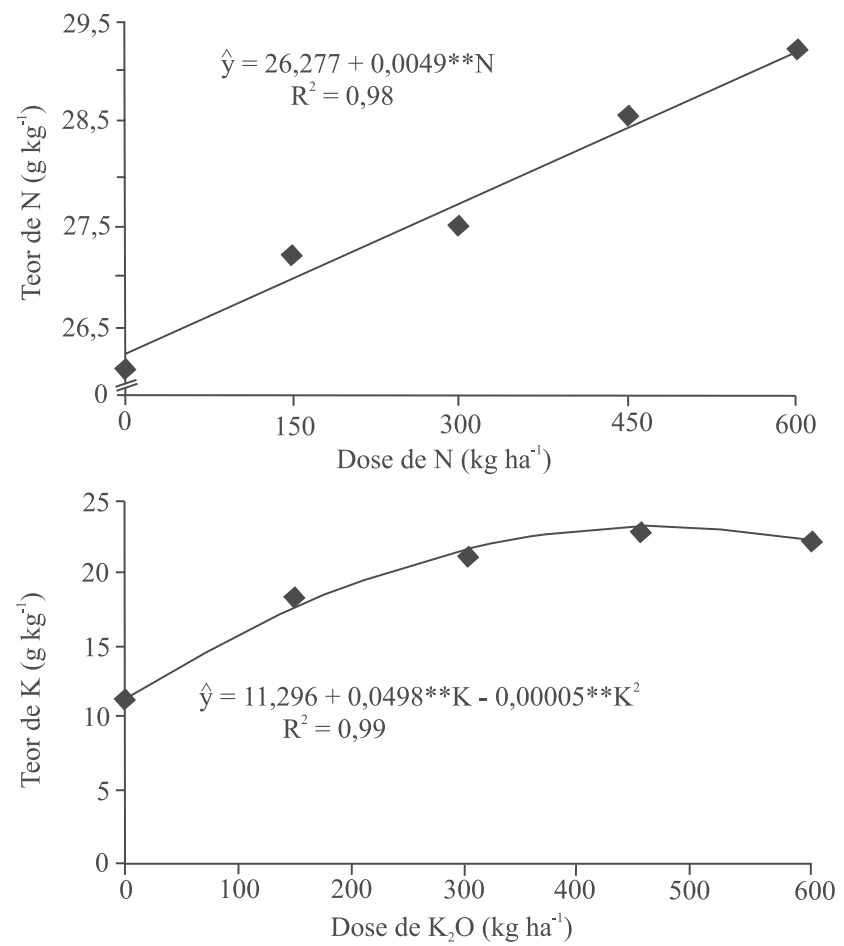

Figura 2. Teores de $\mathrm{N}$ e $\mathrm{K}$ nas folhas de café após a colheita, no sexto ano de cultivo (experimento 2 ). **Significativo a $1 \%$ de probabilidade. 
proporcionou menor bienalidade de produção. Além disso, nos tratamentos em que houve fertilização, a produtividade variou em patamares mais elevados, uma vez que os valores absolutos de produtividade foram muito maiores nesses tratamentos, conforme já relatado (Tabela 2).

À semelhança do primeiro experimento, a variabilidade produtiva de cada parcela ao longo do tempo também foi influenciada pelos tratamentos, no segundo experimento.

$\mathrm{Na}$ análise da bienalidade por meio dos coeficientes de variação, a relação entre adubação e maior estabilidade produtiva pode ser atribuída, essencialmente, à adubação nitrogenada (Figura 4), uma vez que o efeito de $\mathrm{K}$ não foi significativo na

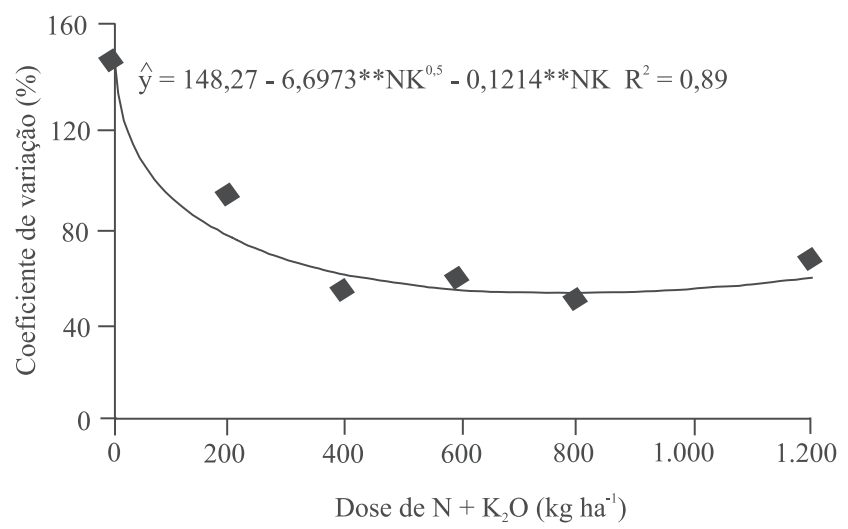

Figura 3. Efeito da adubação com $\mathrm{N}+\mathrm{K}_{2} \mathrm{O}$ (aplicados na proporção 1:1) sobre a bienalidade da produção (\%) do cafeeiro (média de oito safras, no experimento 1). **Significativo a $1 \%$ de probabilidade.

Tabela 3. Bienalidade da produção do cafeeiro ${ }^{(1)}$ em função das doses de $\mathrm{N}+\mathrm{K}\left(\mathrm{kg} \mathrm{ha}^{-1}\right.$ de $\mathrm{N}+\mathrm{K}_{2} \mathrm{O}$, aplicados na proporção 1:1), na transição dos anos de alta para baixa produtividade e dos de baixa para alta.

\begin{tabular}{lcc}
\hline Biênio & Equação & $\mathrm{R}^{2}$ \\
\hline & Alta para baixa produtividade \\
$2002 / 2003-2003 / 2004$ & $\hat{\mathrm{y}}=91,607-1,616^{* *} \mathrm{x}^{0,5}+0,0438^{* *} \mathrm{x}$ & 0,43 \\
$2004 / 2005-2005 / 2006$ & $\hat{\mathrm{y}}=54,76-0,057 \mathrm{x}+0,00004 \mathrm{x}^{2}$ & 0,41 \\
$2006 / 2007-2007 / 2008$ & $\hat{\mathrm{y}}=72,04-2,669^{* *} \mathrm{x}^{0,5}+0,0969 \mathrm{x}$ & 0,67 \\
$2008 / 2009-2009 / 2010$ & $\hat{\mathrm{y}}=\overline{\mathrm{y}}=82$ & - \\
\hline & Baixa para alta produtividade \\
$2003 / 2004-2004 / 2005$ & $\hat{\mathrm{y}}=\overline{\mathrm{y}}=324$ & - \\
$2005 / 2006-2006 / 2007$ & $\hat{\mathrm{y}}=1.986,61-163,264 * * \mathrm{x}^{0,5}+3,236^{* *} \mathrm{x}$ & 0,96 \\
$2007 / 2008-2008 / 2009$ & $\hat{\mathrm{y}}=812,83-2,669^{* *} \mathrm{x}^{0,5}+0,0484 * \mathrm{x}$ & 0,98 \\
\hline
\end{tabular}

(1)Bienalidade nos anos de alta para baixa produtividade $(\%)=[(\mathrm{Pa} \mathrm{Pb}) / \mathrm{Pa}] \times 100$, em que: $\mathrm{Pa}$ é a produtividade no ano de alta e $\mathrm{Pb}$ é a produtividade no ano de baixa; e bienalidade nos anos de baixa para alta $(\%)=[(\mathrm{Pa} \mathrm{Pb}) / \mathrm{Pb}] \times 100$. * e**Significativo a 5 e $1 \%$ de probabilidade, respectivamente. análise geral (não segmentada em biênios). Aqui, é importante lembrar que o requerimento de $\mathrm{N}$ pelo cafeeiro adensado é crescente ao longo dos anos (Figura 1), enquanto o requerimento por K acompanha as flutuações da produção de frutos, pelo menos nos primeiros anos de produção. O cafeeiro produz flores no ramo do ano anterior, o que torna sua produção dependente de fatores relacionados ao crescimento vegetativo da planta, entre os quais o $\mathrm{N}$ tem grande importância (Nazareno et al., 2003; Da Matta et al., 2007).

$\mathrm{Na}$ análise segmentada em biênios, constatouse que, na transição de anos de alta para baixa produtividade de frutos, o $\mathrm{N}$ esteve associado à redução da bienalidade da produção do cafeeiro em dois dos três biênios estudados (Tabela 4), enquanto o K não esteve associado a essa variação em nenhum desses três biênios. No entanto, na transição dos anos de baixa para alta produtividade, no segundo biênio,

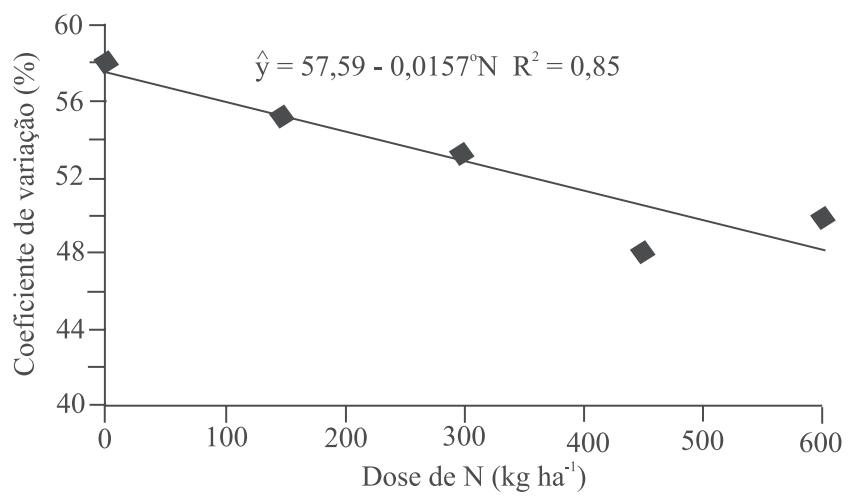

Figura 4. Efeito da adubação com $\mathrm{N}$ sobre a bienalidade da produção do cafeeiro (média de 6 safras, no experimento 2). oSignificativo a $10 \%$ de probabilidade.

Tabela 4. Bienalidade da produção do cafeeiro ${ }^{(1)}$ em função das doses individuais de $\mathrm{N}$ e de $\mathrm{K}\left(\mathrm{kg} \mathrm{ha}^{-1}\right.$ de $\mathrm{N}$ e de $\mathrm{K}_{2} \mathrm{O}$, aplicados separadamente), na transição dos anos de alta para baixa produtividade e dos de baixa para alta.

\begin{tabular}{|c|c|c|}
\hline Biênio & Equação & $\mathrm{R}^{2}$ \\
\hline \multicolumn{3}{|c|}{ Alta para baixa produtividade } \\
\hline $2005 / 2006-2006 / 2007$ & $\hat{\mathrm{y}}=53,284-2,3499 * \mathrm{~N}^{0,5}+0,0634 \mathrm{~N}$ & 0,74 \\
\hline $2007 / 2008-2008 / 2009$ & $\hat{y}=\bar{y}=70$ & \\
\hline 2009/2010-2010/2011 & $\hat{\mathrm{y}}=55,236-0,0272 * \mathrm{~N}$ & 0,74 \\
\hline \multicolumn{3}{|c|}{ Baixa para alta produtividade } \\
\hline $2006 / 2007-2007 / 2008$ & $\hat{\mathrm{y}}=\overline{\mathrm{y}}=55$ & \\
\hline 2008/2009-2009/2010 & $\hat{\mathrm{y}}=37,796+1,9472 \mathrm{~K}^{0,5}-0,0609 \mathrm{~K}$ & 0,94 \\
\hline
\end{tabular}

Pesq. agropec. bras., Brasília, v.48, n.3, p.296-303, mar. 2013 DOI: $10.1590 / \mathrm{S} 0100-204 \mathrm{X} 2013000300008$ 
a adição de $\mathrm{K}$ proporcionou aumento do efeito bienal positivo. Isso significa que a adição de $\mathrm{K}$ relacionou-se a um maior incremento relativo de produtividade nos anos de alta, em comparação aos anos de baixa carga de frutos. Este resultado difere dos obtidos no primeiro experimento, em que a adubação com $\mathrm{N}$ e K reduziu a bienalidade de produção, não importa se positiva (nos anos de baixa para alta) ou negativa (nos anos de alta para baixa). Uma vez que os tratamentos não variaram de um ano para o outro - a dose de $\mathrm{K}$ foi a mesma em cada tratamento, independentemente se em ano de baixa ou de alta -, a maior bienalidade positiva observada com as maiores doses de $\mathrm{K}$ indica que este resultado também pode estar associado à adubação com $\mathrm{K}$ no ano de baixa.

O possível efeito da fertilização realizada na safra anterior sobre a produtividade da safra seguinte encontraria suporte no fato de a nutrição mineral das plantas afetar todas as suas funções vitais e não exclusivamente aspectos relacionados à reprodução. Portanto, por mais que a demanda nutricional varie ao longo dos anos, especialmente quanto ao $\mathrm{K}$, as fertilizações não devem ser formuladas apenas para o atendimento das necessidades nutricionais associadas à carga pendente de frutos, pois essa prática poderia comprometer a produção na safra seguinte, o que está de acordo com Malavolta et al. (2002).

\section{Conclusões}

1. As maiores respostas a $\mathrm{N}$ em relação a $\mathrm{K}$ indicam que a generalização das formulações tradicionais, com esses nutrientes em igual proporção, pode resultar em fertilizações desequilibradas e no desperdício de fertilizantes em cafezais adensados.

2. A adubação com $\mathrm{N}$ reduz a bienalidade de produção do cafeeiro.

3. A adubação com $\mathrm{K}$, em anos de baixa produtividade, pode favorecer a recuperação da produtividade do cafeeiro no ano seguinte.

\section{Referências}

ALVAREZ V., V.H.; NOVAIS, R.F.; BARROS, N.F.; CANTARUTTI, R.B.; LOPES, A.S. Interpretação dos resultados das análises de solos. In: RIBEIRO, A.C.; GUIMARÃES, P.T.G.; ALVAREZ V., V.H. (Ed.). Recomendações para uso de corretivos e fertilizantes em Minas Gerais - $5^{\text {a }}$ aproximação. Viçosa: Comissão de Fertilidade do Solo do Estado de Minas Gerais, 1999. p.13-20.
AUGUSTO, H.S.; MARTINEZ, H.E.P.; SAMPAIO, N.F.; CRUZ, C.D.; PEDROSA, A.W. Concentração foliar de nutrientes em cultivares de Coffea arabica L. sob espaçamentos adensados. Ciência e Agrotecnologia, v.31, p.973-981, 2007. DOI: 10.1590/ S1413-70542007000400005

BRACCINI, M. do C.L.; BRACCINI, A. de L. e; SCAPIM, C.A.; VIDGAL FILHO, P.S.; ZABINI, A.V. Produção de grãos, concentração e aproveitamento de nutrientes em resposta ao aumento na densidade de plantio do cafeeiro. Acta Scientiarum. Agronomy, v.24, p.1205-1211, 2002.

BRAGANÇA, S.M.; SILVA, E.B.; MARTINS, A.G.; SANTOS, L.B.; LANI, J.A.; VOLPI, P.S. Resposta do cafeeiro conilon à adubação de NPK em sistema de plantio adensado. Coffee Science, v.4, p.67-75, 2009.

CANTARUTTI, R.B.; ALVAREZ V., V.H.; RIBEIRO, A.C. Amostragem de solo. In: RIBEIRO, A.C.; GUIMARÃES, P.T.G.; ALVAREZ V., V.H. (Ed.). Recomendações para uso de corretivos e fertilizantes em Minas Gerais - $5^{\text {a }}$ aproximação. Viçosa: Comissão de Fertilidade do Solo do Estado de Minas Gerais, 1999. p.13-20.

DA MATTA, F.M.; RENA, A.B. Ecofisiologia de cafezais sombreados e a pleno Sol. In: ZAMBOLIM, L. (Ed.). O estado da arte de tecnologias na produção de café. Viçosa: UFV, 2002. p.93-135.

DA MATTA, F.M.; RONCHI, C.P.; MAESTRI, M.; BARROS, R.S. Ecophysiology of coffee growth and production. Brazilian Journal of Plant Physiology, v.19, p.485-510, 2007. DOI: 10.1590/S1677-04202007000400014.

FIGUEIREDO, F.C.; FURTINI NETO, A.E.; GUIMARÃES, P.T.G.; SILVA, E. de B.; BOTREL, P.P. Eficiência da adubação com NPK na produção de cafezais adensados na região Sul de Minas Gerais. Coffee Science, v.1, p.135-142, 2006.

GALLO, P.B.; RAIJ, B.V.; QUAGGIO, J.A.; PEREIRA, L.C.E. Resposta de cafezais adensados à adubação NPK. Bragantia, v.58, p.341-351, 1999. DOI: 10.1590/ S0006-87051999000200014.

GUIMARÃES, P.T.G.; GARCIA, A.W.R.; ALVAREZ VIEGAS, V.H.; PREZOTTI, L.C.; VIANA, A.S.; MIGUEL, A.E.; MALAVOLTA, E.; CORREAA, J.B.; LOPES, A.S.; NOGUEIRA, F.D.; MONTEIRO, A.V.C. Cafeeiro. In: RIBEIRO, A.C.; GUIMARÃES, P.T.G.; ALVAREZ V., V.H. (Ed.). Recomendações para uso de corretivos e fertilizantes em Minas Gerais - $5^{\text {a }}$ aproximação. Viçosa: Comissão de Fertilidade do Solo do Estado de Minas Gerais, 1999. p.13-20.

LAVIOLA, B.G.; MARTINEZ, H.E.P.; SOUZA, R.B.; ALVAREZ V., V.H. Dinâmica de N e K em folhas, flores e frutos de cafeeiro arábico em três níveis de adubação. Bioscience Journal, v.22, p.33-47, 2006.

MALAVOLTA, E.; FAVARIN, J.L.; MALAVOLTA, M.; CABRAL, C.P.; HEINRICHS, R.; SILVEIRA, J.S.M. Repartição de nutrientes nos ramos, folhas e flores do cafeeiro. Pesquisa Agropecuária Brasileira, v.37, p.1017-1022, 2002. DOI: 10.1590/ S0100-204X2002000700016.

NAZARENO, R.B.; OLIVEIRA, C.A. da S.; SANZONOWICZ, C.; SAMPAIO, J.B.R.; SILVA, J.C.P. da; GUERRA, A.F. 
Crescimento inicial do cafeeiro Rubi em resposta a doses de nitrogênio, fósforo e potássio e a regimes hídricos. Pesquisa Agropecuária Brasileira, v.38, p.903-910, 2003. DOI: 10.1590/ S0100-204X2003000800002.

NEVES, Y.P.; MARTINEZ, H.E.P.; AMARAL, J.F.T. do; SOUZA, R.B. de; DOMINGOS, D.R. Produtividad y acumulación de materia seca, N, P y K por cultivares de Coffea arabica L. Coffee Science, v.2, p.156-167, 2006.

PAVAN, M.A.; CHAVES, J.C.D.; ANDROCIOLI FILHO, A. Produção de café em função da densidade de plantio, adubação e tratamento fitossanitário. Turrialba, v.44, p.227-231, 1994.

PEREIRA, S.P.; BARTHOLO, G.F.; BALIZA, D.P.; SOBREIRA, F.M.; GUIMARÃES, R.J. Crescimento, produtividade e bienalidade do cafeeiro em função do espaçamento de cultivo. Pesquisa Agropecuária Brasileira, v.46, p.152-160, 2011. DOI: 10.1590/S0100-204X2011000200006.

PREZOTTI, L.C.; ROCHA, A.C. da. Nutrição do cafeeiro arábica em função da densidade de plantas e da fertilização com NPK. Bragantia, v.63, p.239-251, 2004. DOI: 10.1590/ S0006-87052004000200009.
RÖMHELD, V.; KIRKBY, E.A. Research on potassium in agriculture: needs and prospects. Plant and Soil, v.335, p.155-180, 2010. DOI: $10.1007 / \mathrm{s} 11104-010-0520-1$.

SANTOS, H.G. dos; JACOMINE, P.K.T.; ANJOS, L.H.C. dos; OLIVEIRA, V.A. de; OLIVEIRA, J.B. de; COELHO, M.R.; LUMBRERAS, J.F.; CUNHA, T.J.F. (Ed.). Sistema brasileiro de classificação de solos. 2.ed. Rio de janeiro: Embrapa Solos, 2006. 306p.

SOBREIRA, F.M.; GUIMARÃES, R.J.; COLOMBO, A.; SCALCO, S.M.; CARVALHO, J.G. Adubação nitrogenada e potássica de cafeeiro fertirrigado na fase de formação, em plantio adensado. Pesquisa Agropecuária Brasileira, v.46, p.9-16, 2011. DOI: 10.1590/S0100-204X2011000100002.

SOUZA, T.R. de; VILLAS BÔAS, R.L.; QUAGGIO, J.A.; SALOMÃO, L.C.; FORATO, L.C. Dinâmica de nutrientes na solução do solo em pomar fertirrigado de citros. Pesquisa Agropecuária Brasileira, v.47, p.846-854, 2012. DOI: 10.1590/ S0100-204X2012000600016.

TSAY, Y.-F.; HO, C.-H.; CHEN, H.-Y.; LIN, S.H. Integration of nitrogen and potassium signaling. Annual Review of Plant Biology, v.62, p.207-226, 2011. DOI: 10.1146/ annurev-arplant-042110-103837.

Recebido em 29 de agosto de 2012 e aprovado em 14 de fevereiro de 2013 


\section{ERRATA}

No artigo, VALADARES, S.V.; NEVES, J.C.L.; ROSA, G.N.G.P.; MARTINEZ, H.E.P.; ALVAREZ V., V.H.; LIMA, P.C. de. Produtividade e bienalidade da produção de cafezais adensados, sob diferentes doses de N e K. Pesquisa Agropecuária Brasileira, v.48, p.296-303, 2013. DOI: 10.1590/S0100204X2013000300008, devem ser feitas as seguintes correções:

No gráfico superior da Figura 2, na legenda do eixo x, onde se lê "“", leia-se "Dose de N (kg ha"-1)".

No gráfico inferior da Figura 2, na legenda do eixo y, onde se lê "Teor de N $\left(\mathrm{g} \mathrm{kg}^{-1}\right)$ ", leia-se "Teor de $\mathrm{K}\left(\mathrm{g} \mathrm{kg}^{-1}\right)$ '.

\section{ERRATA}

In the paper "VALADARES, S.V.; NEVES, J.C.L.; ROSA, G.N.G.P.; MARTINEZ, H.E.P.;

ALVAREZ V., V.H.; LIMA, P.C. de. Produtividade e bienalidade da produção de cafezais adensados, sob diferentes doses de N e K. Pesquisa Agropecuária Brasileira, v.48, p.296-303, 2013. DOI:

10.1590/S0100-204X2013000300008", the following corrections should be done:

In the upper graph of Figure 2, in the legend on X-axis, where it reads "", it should read "Dose de $\mathrm{N}$ $\left(\mathrm{kg} \mathrm{ha}^{-1}\right)^{\prime \prime}$.

In the lower graph of Figure 2, in the legend on the y-axis, where it reads "Teor de N $\left(\mathrm{g} \mathrm{kg}^{-1}\right)$ ", it should read "Teor de K $\left(\mathrm{g} \mathrm{kg}^{-1}\right)$ ". 